\title{
Responding under single vs. multiple schedules
}

\author{
ROBERT W. POWELL \\ University of South Florida, Tampa, Florida 33620
}

\begin{abstract}
Responding under variable-ratio and variable-interval schedules was compared in pigeons when the schedules were presented singly or as components in a multiple schedule. All birds responded at higher rates under the variable-ratio schedule, even though rate of reinforcement was usually higher under the variable-interval schedule. Presenting these two schedules as components in a multiple schedule and varying the duration of these components appeared to produce no appreciable effect upon the rate or pattern of responding under either schedule, in comparison with single-schedule performance. A second experiment employed the same procedures to compare responding under variable-ratio and fixed-ratio schedules. Variable-ratio responding was again higher, but this was mainly a function of consistent postreinforcement pauses under the fixed-ratio schedules, which reduced overall response rate. A notable interaction effect was observed here, in that postreinforcement pauses were much shorter and running rate (postreinforcement pauses excluded) was lower under the fixed-ratio schedule when it was presented as part of a multiple schedule, in comparison with performance under the single fixed-ratio schedule. Variations in the duration of the multiple-schedule components had no discernible effects upon performance under either schedule.
\end{abstract}

Multiple (mult) schedules involve two or more independent schedules of reinforcement that are presented successively to the subject, with each schedule signaled by a different stimulus. A number of psychologists see the mult schedule as providing an elegant control procedure, in that it allows one to study the effects of different contingencies on the behavior of a single subject within a brief period of time (one experimental session). A potential pitfall for this type of analysis, however, resides in the frequent observation of interaction effects under mult schedules (Catania, 1961; Reynolds, 1961a, 1961b). Many researchers have found that the contingency in effect under one component of a mult schedule will also influence responding under the other component, particularly when there is a change in the first contingency. It appears that most researchers have simply assumed that the behavior generated under a particular contingency (e.g., a fixed-ratio, FR, schedule) as part of a mult schedule would be quite similar to the behavior generated by the same contingency presented as a single schedule. The present research was undertaken as a descriptive study intended to assess the validity of this assumption. This study also examined the effect of changes in component duration under the mult schedule.

\section{EXPERIMENT 1: COMPARISON OF SINGLE AND MULT VARIABLE-RATIO (VR) AND VARIABLE- INTERVAL (VI) SCHEDULES}

While most researchers have found that VR schedules

Reprints may be obtained from Robert W. Powell, Department of Psychology, University of South Florida, Tampa, Florida 33620. produce higher response rates than VI schedules (Ferster \& Skinner, 1957; Herrnstein, 1964; Powell, 1970, 1971), the controlling variables have been difficult to specify. A major problem resides in the fact that interval schedules directly control rate of reinforcement, but probability of reinforcement varies in relation to the subject's response rate. Exactly the opposite is true for ratio schedules. This has led some theorists to conclude that schedules of reinforcement are too complex to permit a valid functional analysis of the controlling variables at this time (Mackintosh, 1974, p. 181-182).

\section{Method}

Subjects. Five adult White Carneaux pigeons were maintained within $10 \mathrm{~g}$ of $75 \%$ of their free-feeding weights. Three pigeons $(16,20,21)$ were experimentally naive, and the others $(30,45)$ had previous FR training.

Apparatus. A Lehigh Valley pigeon test chamber (Model 1519C) was employed. Mixed grain was used for reinforcement. Reinforcement time was 3.0 sec for each bird. During reinforcement, the key light was turned off. Data were recorded by digital counters and a Gerbrands cumulative recorder.

Procedure. Single schedules. Following shaping in the presence of a white key light, the previously naive pigeons $(16,20,21)$ were exposed to gradually extended VR and VI schedules, which were presented during alternate sessions. A green key light was always present during VR sessions, and the VI schedules were signaled by a red key light. Training continued until stable performance was achieved under both VR 100 and VI 1-min schedules, which were the terminal schedules employed in this experiment. These values were selected with the expectation, based on prior experience, that they would produce approximately equal rates of reinforcement. The VR schedule included 18 terms, ranging from 5 to 210; the VI schedule included 12 terms, ranging from 2 to 170 sec. The same procedure was followed with the previously conditioned birds $(30,45)$, except that the terminal schedules were in effect throughout training. Training sessions were usually $30 \mathrm{~min}$ in duration for all birds. The number of training sessions ranged from 42 (Bird 16) to 54 (Bird 30). After stable performance was achieved, as judged 
by inspection of cumulative records, the five birds were studied for 10 additional 30 -min sessions, with each of the terminal schedules present during alternate sessions.

Mult schedules. All birds were then studied under mult schedules in which the VR and VI components alternated every $6 \mathrm{~min}$. All experimental sessions were $36 \mathrm{~min}$ in duration, with three components each of VR and VI per session. In addition, the sequence was alternated so that a VR component was presented first one day and a VI component the next. Three of the birds $(16,20,21)$ were studied for 25 sessions under this procedure, and the remaining two birds $(30,45)$ were each studied for 10 sessions.

Changes in component duration. The effects of changes in component duration were studied in three birds $(21,30,45)$. Series of 10 sessions under single VR and VI schedules alternated with 10 sessions under mult VR-VI schedules. During each successive series of mult schedule sessions, the component duration was changed. Twenty additional mult schedule sessions were conducted for Bird 45, because of changes that developed in response rate. Experimental session were $36 \mathrm{~min}$ in duration.

\section{Results}

The VR schedule produced higher response rates in every bird under both single and mult schedules. The frequency of reinforcement was approximately equal under the two schedules for Birds 16 and 20 and was slightly higher, under the VI schedule, for the remaining birds. These data are summarized in Figure 1. No consistent interaction effects were produced by the change from single to mult schedules. While changes in response rates did occur, there was no uniformity for different birds in the direction of change under either schedule.

The changes in component duration produced no consistent effect upon response rates, as indicated by the data summarized in Figure 2. The VR rates remained consistently higher, except for a long-term convergence of the rates of Bird 45. This convergence does not appear to be a function of component duration, however, in that the difference in rates under the single schedules also grew progressively smaller. A marked divergence in the response rates of Bird 30 occurred, but again the trend was present to the same extent under both single and mult schedules. The VI rates remained more stable than the VR rates for Birds 30 and 45, whereas the response rates for Bird 21 were equally consistent under each schedule.

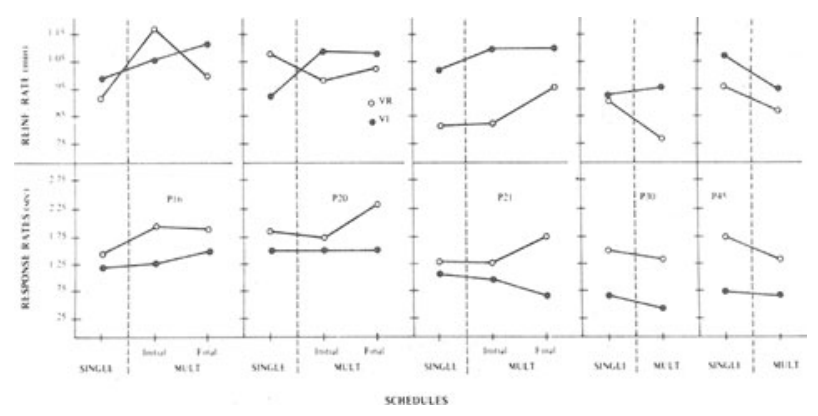

Figure 1. Response rates and reinforcement rates for each pigeon under the VR and VI schedules with the change from single to mult schedules. Each data point represents the mean performance over 10 sessions.

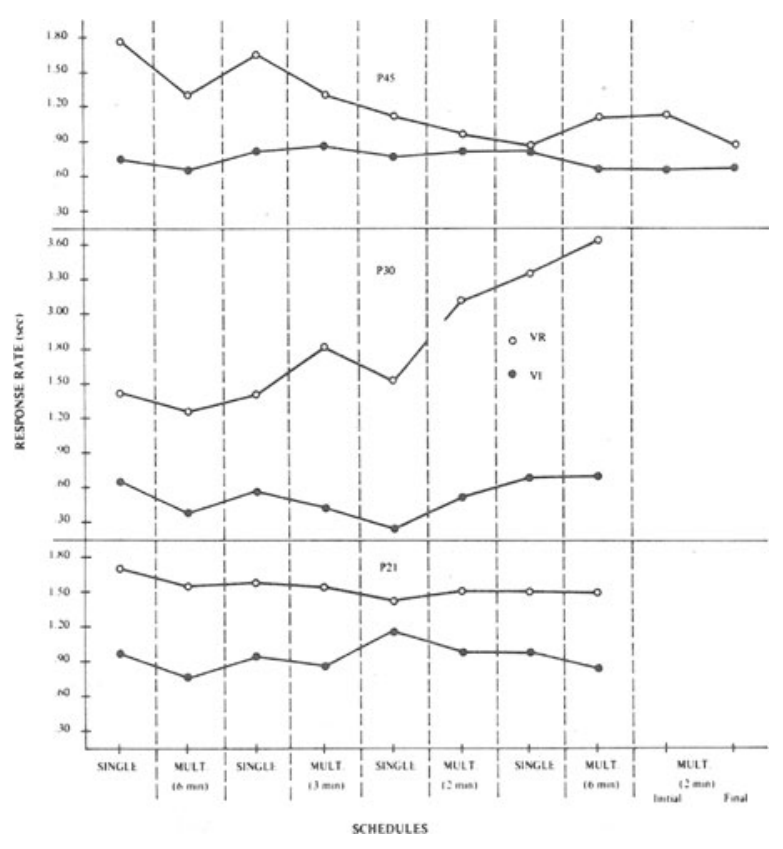

Figure 2. Response rates under single- and mult-schedule VR and VI sessions as the component durations were changed with each successive series of mult-schedule sessions. Each data point represents the mean performance over 10 sessions.

\section{EXPERIMENT 2: COMPARISON OF SINGLE AND MULT VR AND FR SCHEDULES}

Fantino (1967) found that a stimulus correlated with mixed-ratio (MR) schedules maintained a higher rate of responding than a stimulus correlated with an FR schedule when the arithmetic mean of the mixed ratios equaled the fixed ratio. MR schedules differ from conventional VR schedules in that only a small number of ratio values compose the MR, whereas a large number (usually 20 or more) are presented in VR schedules.

The present experiment was conducted to study response rates under VR and FR schedules with equal response requirements, on the average. In studies of FR behavior, it has become common practice to consider separately the postreinforcement pause (PRP) and the rate from the first response through the last in the ratio. PRP refers to the latency of the first response after reinforcement. The practice of analyzing these dependent measures separately is empirically based, for consistent relationships have been found between pausing and independent variables such as ratio size (Felton \& Lyon, 1966; Powell, 1968), reinforcement magnitude (Powell, 1969b), and deprivation (Malott, 1966; Powell, 1969a). These same studies found no consistent relationship between the variables studied and the FR running rate. In the present experiment, FR response rates were calculated with the PRPs included in some cases and excluded in others. For purposes of clarity, overall FR response rate refers to analyses in which pauses are included, and FR running rate refers to analyses in which pauses are excluded. 


\section{Method}

Subjects. Six additional White Carneaux pigeons were studied $(26,33,41,43,44,50)$. All birds had previous FR training. They were maintained within $\pm 10 \mathrm{~g}$ of $75 \%$ of their free-feeding weights.

Apparatus. The apparatus was the same as that used in Experiment 1, except that the elapsed time from the end of the reinforcement period to the first response in the ratio (i.e., the PRP) was measured under the FR schedule. Pause durations were recorded individually by a Grason-Stadler printout counter, as well as cumulatively over each daily session.

Procedure. Single schedules. The terminal schedules were VR 100 and FR 100, with VR signaled by a green key light and FR signaled by a white key light. Birds 33,44 , and 50 were studied under this procedure, and each was exposed to the terminal schedules for approximately 50 training sessions of 30 min duration prior to 10 test sessions, with the VR and FR schedules present on alternate days.

Mult schedules. Birds 33, 44, and 50 were then studied for 20 mult-schedule sessions of 36 min duration, with the terminal schedules in effect. Components in the mult schedule alternated every $6 \mathrm{~min}$.

Changes in component duration. Birds 26,41 , and 43 were studied following approximately 50 sessions training under the terminal VR and FR schedules. These sessions were generally 30 min long. For Birds 41 and 43, the same sequence was used as in Experiment 1, except that the last step was omitted. Steps 2 and 3 were omitted for Bird 26, and then the same sequence was followed as for the other two birds. All sessions were $36 \mathrm{~min}$ in duration.

\section{Results}

The VR schedule again produced the highest response rates in every case, except for the performance of Bird 50 under single schedules. Figure 3 shows that the rates for Birds 33 and 44 did not change substantially with the switch from single to mult schedules, whereas the rates for Bird 50 changed from a higher overall FR response rate when only single schedules were presented to a higher rate under VR during mult-schedule sessions. Inspection of cumulative records under the mult-schedule revealed that the FR schedule produced reliable pauses after reinforcement, but the VR schedule did not.

When changes in component duration were made for Birds 26,41 , and 43 , no consistent effect upon response rates under the two schedules was found. Figure 4 pre-

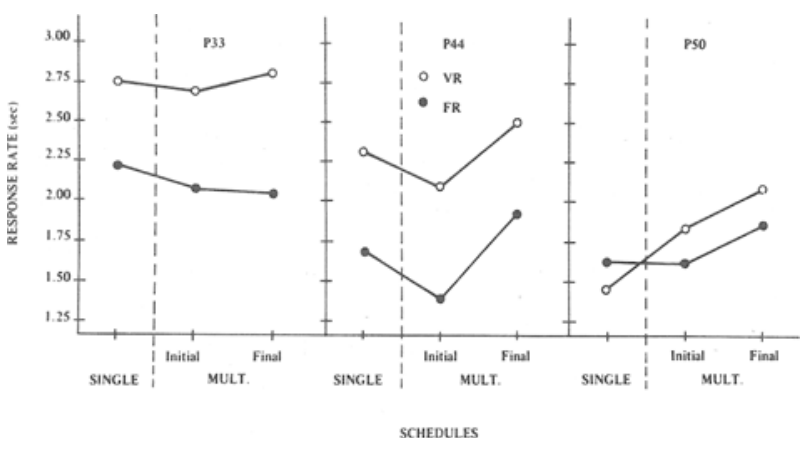

Figure 3. Response rates for three pigeons under the VR and FR schedules with the change from single to mult schedules. Each data point represents the mean performance over 10 sessions.

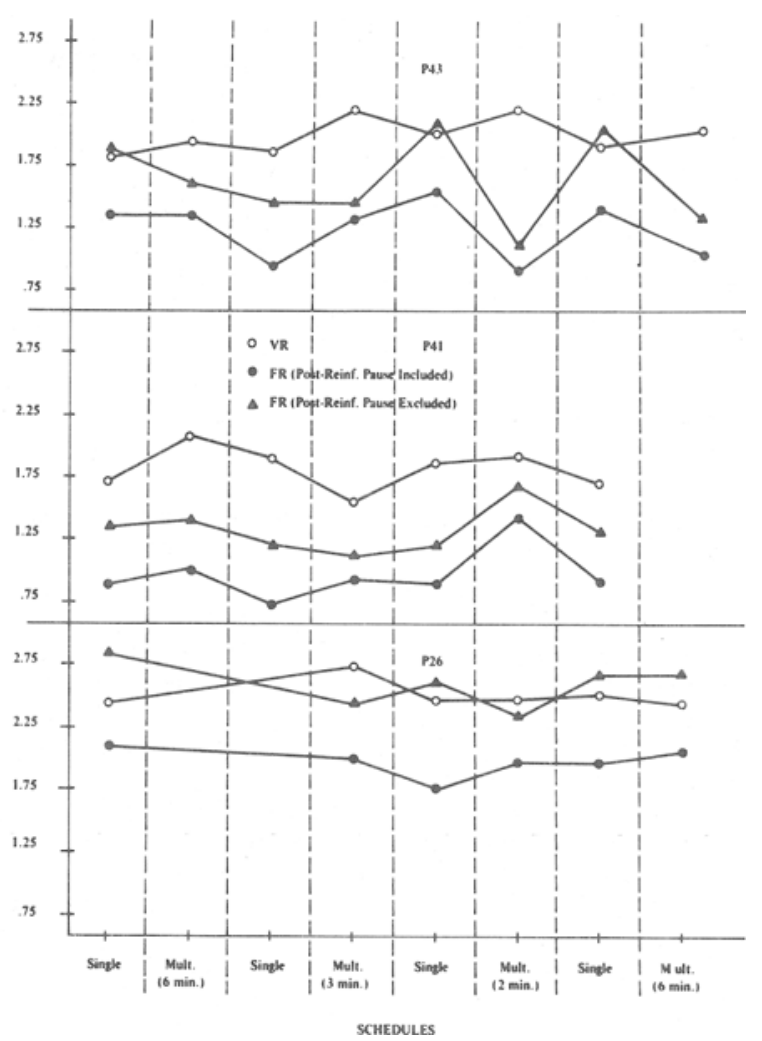

Figure 4. Response rates under single- and mult-schedule VR and FR sessions as the component durations were changed with each successive series of mult-schedule sessions. Both overall FR response rates (PRPs included) and FR running rates (PRPs excluded) are presented. Each data point represents the mean performance over 10 sessions.

sents both the overall FR response rate (PRP included) and the FR running rate (PRP excluded), in addition to the VR response rate. While the VR rate was consistently higher than the overall FR rate for all birds, the FR running rate sometimes exceeded the VR response rate for Birds 26 and 43 . This occurred seven times, six of which involved single schedules.

An analysis of PRPs with changes in component duration is presented in Figure 5. These data show that the mean PRP was much shorter for all birds on sessions in which FR was part of a mult schedule than on sessions in which only FR was presented. The mean PRPs in seconds were 15.6 and 9.5 (Bird 26), 42.7 and 15.3 (Bird 41), and 25.3 and 15.7 (Bird 43) under the single and mult schedules, respectively. Changes in component duration had no systematic effect upon the PRP.

\section{DISCUSSION}

A VR 100 schedule produced higher response rates in each of five pigeons than did a VI 1-min schedule, even though the rate of reinforcement was generally higher under the VI schedule. When these schedules were presented as components in a mult schedule, both the rate and pattern of responding appeared to 


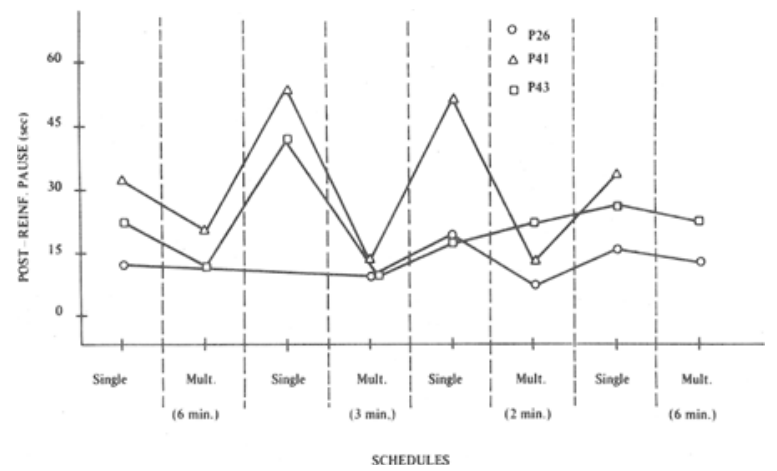

Figure 5. PRPs under the FR schedules during single- and mult-schedule sessions. Each data point represents the mean performance over 10 sessions.

be unchanged from what it had been under the respective single schedules. That is, there was no evidence of interaction between the mult-schedule components. Variation in the duration of these components also failed to produce discernible effects upon the responding generated by these two schedules. VR response rates continued to be higher under these manipulations.

When this VR 100 schedule was compared with an FR 100 schedule in six additional pigeons, five birds showed substantially higher response rates under the VR schedule whether it was presented singly or as part of a mult schedule. However, this difference was largely attributable to the PRP that occurs under FR but not under VR schedules. When the PRP was excluded in calculating the response rate under FR (FR running rate), response rates under VR were somewhat higher under mult schedules, but under single schedules, there was little or no difference between VR response rate and FR running rate. Correspondingly, FR running rate was somewhat lower under the mult FR-VR schedule than it was under the single FR schedule. At the same time, PRPs under FR were much shorter under the mult FR-VR schedule than they were under the single FR schedule. Thus it would appear that interaction effects did take place under the mult schedule, being most obvious in relation to changes in FR behavior. While the decrease in PRPs under FR can be plausibly attributed to generalization or positive induction from the VR schedule, the decrease in FR running rate can not be similarly explained. Rather, it would appear that the FR behavior was influenced as a unit, with PRPs decreasing and running rate decreasing, so that the overall response rate remained essentially the same as under the single FR schedule. Changes in component duration appeared to have no systematic effects upon the mult VR-FR schedule.

\section{REFERENCES}

Catania, A. C. Behavioral contrast in a mult. and concurrent schedule of reinforcement. Journal of the Experimental Analysis of Behavior, 1961, 4, 335-342.

Fantino, E. Preference for mixed-ratio vs fixed-ratio schedules. Journal of the Experimental Analysis of Behavior, 1967, 10, 35-43.

Felton, M., \& Lyon, D. O. The post-reinforcement pause. Journal of the Experimental Analysis of Behavior, 1966, 9, 131-134.

Ferster, C. B., \& Skinner, B. F. Schedules of reinforcement. New York: Appleton-Century-Crofts, 1957.

HERRNSTE IN, R. J. Secondary reinforcement and rate of primary reinforcement. Journal of the Experimental Analysis of Behavior, 1964, 7, 27-36.

Mackintosh, N. J. The psychology of animal learning. London: Academic Press, 1974.

Malot, $R$. W. The effects of prefeeding in plain and chained fixed-ratio schedules of reinforcement. Psychonomic Science, 1966, 4, 285-286.

Powell, R. W. The effect of small sequential changes in fixedratio size upon the post-reinforcement pause. Journal of the Experimental Analysis of Behavior, 1968, 11, 589-593.

Powell, R. W. The effect of deprivation upon fixed-ratio responding. Psychonomic Science, 1969, 16, 17-18. (a)

Powell, R. W. The effect of reinforcement magnitude upon responding under fixed-ratio schedules. Journal of the Experimental Analysis of Behavior, 1969, 12, 605-608. (b)

Powell, R. W. The effect of punishment shock intensity upon responding under multiple schedules. Journal of the Experimental Analysis of Behavior, 1970, 14, 201-211.

Powell, R. W. Some effects of punishment shock intensity upon discrimination responding. Journal of the Experimental Analysis of Behavior, 1971, 15, 109-116.

REYNolds, G. A. An analysis of interactions in a multiple schedule. Journal of the Experimental Analysis of Behavior, 1961, 4, 107-117. (a)

Reynolds, G. S. Behavioral contrast. Journal of the Experimental Analysis of Behavior, 1961, 4, 57-71. (b)

(Received for publication October 24, 1980.) 